\title{
Pour une autonomie relative des niveaux sémantique, énonciatif et iconique des textes poétiques
}

\author{
Monte Michèle \\ Université Lumière Lyon 2, ICAR, UMR 5191 \\ michele.monte@univ-lyon2.fr
}

L'année 2011 a été marquée par deux parutions importantes pour qui s'intéresse à l'approche linguistique de la poésie : Baudelaire, les notes préparatoires rédigées par Benveniste en 1967 en vue d'un article qui n'a finalement pas vu le jour, et Poétique de l'évocation qui constitue la synthèse longtemps attendue des travaux de Marc Dominicy. La confrontation de ces études séparées par une trentaine d'années nous permet de mesurer combien les développements récents de la linguistique, dans les domaines de la sémantique et de l'analyse des textes et des discours, ont renouvelé la vieille question de la spécificité du discours poétique. Prendre acte de ce qui est devenu caduc tout en reconnaissant les apports de nos devanciers et les pistes qu'ils ouvrent parfois à leur insu me parait important dans une discipline qui a tendance tantôt à douter de ses progrès, tantôt à se précipiter sur les idées à la mode en oubliant sa propre tradition.

Mais avant même que l'actualité éditoriale ne remette à l'honneur la réflexion des linguistes sur la poésie, c'est la question des genres et des archi-genres qui a motivé cette contribution, et plus spécifiquement l'insatisfaction que me laissent souvent les approches déshistoricisées de la poésie. Or s'il est un archigenre qui a connu des bouleversements radicaux depuis le XIXe siècle, c'est bien celui-là. Est-il encore possible d'en parler au singulier? et si oui, à quelles conditions ? Pour répondre à cette question, je m'interrogerai tout d'abord sur la façon dont un locuteur ordinaire conceptualise la question de la poésie. Puis, en m'appuyant sur les travaux des poéticiens tels que Genette, Schaeffer ou Guerrero, je retracerai à grands traits la façon dont l'archi-genre " poésie » a conquis sa place dans la catégorisation littéraire et ce qui en est résulté. Je résumerai ensuite les propositions de Jakobson, Ruwet, Benveniste et Dominicy, en dégageant les questions cruciales qu'elles posent quant à une définition linguistique de la poésie. Je terminerai en proposant une approche graduelle et multi-critères de la poésie comme archi-genre dont l'objectif serait de ne pas laisser d'emblée de côté des pans entiers de la production poétique tout en faisant droit également aux évolutions qui se sont manifestées depuis la fin du XIXe siècle.

\section{Que nous apprend la catégorisation spontanée ?}

Je partirai de l'expérience que peut faire un usager du métro parisien à certaines périodes de l'année. Les rames et les couloirs de métro proposent parfois sur leurs espaces d'affichage des poèmes. A quoi l'usager va-t-il les reconnaître comme tels ? Le critère déterminant sera celui que Genette (1991) appelle la diction, soit un ensemble de propriétés formelles : disposition spatiale, c'est-à-dire au minimum retour à la ligne dû à la forme versifiée et parfois éclatement sur la page, récurrences rythmiques et phonétiques, éventuellement rimes, mètres et strophes. Ces critères formels déclencheront chez le lecteur une attitude de réception spécifique : il va considérer le texte comme un objet esthétique qui n'est pas en rapport direct avec sa vie quotidienne et qui requiert une interprétation différente de celle qu'exigent les énoncés qui tapissent ordinairement les couloirs du métro, qu'il s'agisse de messages publicitaires ou de textes informatifs produits par la RATP. Il appréciera le texte à l'aune de deux critères : d'une part, sa capacité à faire surgir en lui des sensations, des pensées ou des émotions qu'il reconnaîtra comme pertinentes pour son existence, d'autre part sa capacité à disposer les mots et les énoncés de façon frappante, amusante ou émouvante. Bien évidemment, d'un lecteur à l'autre, les deux critères n'auront pas le même poids et ce ne seront pas les mêmes éléments qui seront valorisés ou tenus pour quantité négligeable. Si le texte proposé est en prose, le lecteur mettra probablement plus de temps à l'identifier comme poème et il devra pour 
cela non seulement l'opposer aux textes non littéraires qui figurent habituellement dans le métro, mais aussi aux autres textes littéraires en prose. D'autres facteurs formels et sémantiques entreront alors en compte : l'autonomie du texte - opposée à l'incomplétude d'un fragment de récit -, son caractère étrange ou humoristique, ses modalités énonciatives. Selon le type d'instruction scolaire que le lecteur aura reçue, l'identification comme poème pourra échouer. En effet, malgré l'évolution de la poésie depuis le XIXe siècle, la poésie se confond encore le plus souvent dans l'éducation de base avec le vers, pour des raisons en grande partie légitimes.

Supposons à présent que notre passager lise dans le métro un texte en vers l'invitant in fine à acheter un appartement dans une nouvelle résidence ou une crème de beauté qui rendra à sa peau tout son éclat; il est probable qu'après avoir cru d'abord lire un poème, il comprendra qu'il a affaire à un texte publicitaire, ou bien il jugera qu'il s'agit d'un poème publicitaire. Nous avons affaire dans ce cas à une dissociation entre les critères formels et les critères pragmatiques et, quelle que soit l'appellation retenue par le lecteur pour catégoriser le texte, l'attitude de réception sera sans doute hybride, à l'image du texte lui-même : le lecteur se positionnera à la fois par rapport au produit proposé et par rapport à la forme même du texte, écrit pour retenir son attention au-delà du message publicitaire proprement dit.

Si l'on reprend les catégories proposées par Maingueneau (2004) pour définir la scène d'énonciation, on observe ainsi que, lorsque la scène générique et la scénographie convergent, le niveau de la scène englobante n'est pas nécessairement pertinent lors de la catégorisation - l'identification comme poème présuppose en effet l'appartenance au discours littéraire -, mais qu'il le redevient lorsqu'elles divergent : une affiche publicitaire (scène générique) en forme de poème (scénographie) doit être reconnue comme appartenant au discours publicitaire (scène englobante) pour être correctement interprétée. Un poème en prose doit de même être identifié comme littéraire pour bénéficier de la réception que l'on fait habituellement au poème.

C'est sur le fond de ces quelques considérations de base que je me propose à présent d'envisager la façon dont certaines évolutions historiques ont façonné notre appréhension de la poésie (2) et dont certaines théories linguistiques ont posé la question du langage ou discours poétique (3).

\section{De quelques simplifications fâcheuses}

Parler de poésie de nos jours n'a rien à voir avec ce que la notion recouvrait dans l'Antiquité, où toute la production littéraire était en vers, ni même à l'époque classique où le roman était encore marginal et où une bonne partie du théâtre était versifié. Genette (1979) et Combe (1992) ont bien montré comment s'est constituée, en France au moins, une tripartition roman / théâtre / poésie qui, d'une part, laisse de côté toute la littérarité conditionnelle représentée par le genre épistolaire, les essais, l'éloquence et ce que j'appellerai pour faire vite l'écriture personnelle (autobiographie, mémoires, journal), et d'autre part, implique un glissement des modes aux genres, pour reprendre la terminologie de Genette, c'est-à-dire des attitudes énonciatives à des productions effectives régies par des normes sociales. Je me bornerai à reprendre à grands traits cette évolution pour ensuite examiner les potentialités de la répartition actuelle.

On sait que Platon et Aristote, l'un pour la dénigrer, l'autre pour l'exalter, ne s'intéressent qu'à la poésie narrative ou dramatique, d'essence mimétique, et ignorent dans leurs réflexions la poésie lyrique pourtant bien représentée à leur époque. Pendant très longtemps, les genres non mimétiques sont difficilement catégorisés par les Arts poétiques qui se contentent de les énumérer ou de proposer des classements bancals :

L'idée que la poésie lyrique correspond à un type d'énonciation réservée au poète paraît donc, d'après ce que nous savons, complètement étrangère à la pensée antique. (Guerrero, $2000: 61$ )

La triade épique/lyrique/dramatique systématisée par le romantisme commence à émerger dans les traités de la fin de la Renaissance qui, à la suite de la République de Platon, utilisent l'adjectif mélique dérivé du grec melos, «chant», pour ce qu'on appellera plus tard la poésie lyrique : Minturno affecte la poésie mélique au «mode de narration simple [...] quand c'est le poète lui-même qui parle, n'assumant le rôle 
de personne. » (cité par Guerrero 2000 : 89). Scaliger reprend la thématique d'Horace et affecte à la poésie lyrique une série de sujets qui mêlent en réalité une thématique et une visée pragmatique : « louanges, amours, invectives, afflictions, propos de table, reproches, prières pour complaire au génie, exhortations » (ibid. p. 99). Mais les théoriciens des XVI ${ }^{\mathrm{e}}-\mathrm{XVII}{ }^{\mathrm{e}}$ siècles achoppent tous sur la question de la mimésis, car ils pressentent sans toutefois le conceptualiser clairement que la poésie lyrique remet en cause l'approche aristotélicienne des œuvres d'art. Pour la sauver, l'abbé Batteux considère que les sentiments exprimés par les poètes « sont donc, au moins pour partie, des sentiments feints par art " (cité par Genette, 1979 : 37). C'est avec Johann Adolf Schlegel, traducteur de Batteux, que toute référence à l'imitation est abandonnée et que la poésie lyrique commence à être assimilée à l'expression par le poète de ses propres sentiments. Mais, avec Friedrich Schlegel, les modes platoniciens d'énonciation énonciation réservée au poète / énonciation alternée / énonciation réservée aux personnages - sont remplacés par une distinction philosophique qui se superpose à la triade des archi-genres : la forme lyrique est qualifiée de subjective, la forme dramatique d'objective et l'épique de subjective-objective. Comme le dit très justement Genette, le critère de répartition se déplace alors « du plan en principe purement technique de la situation énonciative vers un plan plutôt psychologique et existentiel » (ibid. p. 43), glissement préparé par l'accent mis sur les sentiments au détriment des actions dans les travaux de Batteux, par exemple. Les penseurs postérieurs (Hegel, Staiger) ordonneront différemment la triade en cherchant un principe expliquant l'apparition et la prédominance successive de l'un ou l'autre des archigenres, mais la poésie lyrique restera constamment attachée à la subjectivité et à l'émotion.

Or on assiste parallèlement au recul très net du vers au théâtre, au développement prodigieux du roman et au déclin de la poésie narrative, malgré de beaux exemples comme La Légende des siècles. Poésie et lyrisme apparaissent à tort comme superposables, phénomène accentué en France par l'anathème porté par Mallarmé, puis par Valéry et Breton contre le narratif: Dominique Combe a bien analysé ce processus d'éviction du narratif dans Poésie et récit (1989) et je n'y reviendrai pas. De ce fait la deuxième moitié du $\mathrm{XX}^{\mathrm{e}}$ siècle hérite, notamment dans l'enseignement de la littérature, d'une situation où la triade lyrique/épique/dramatique centrée au départ sur des modes d'énonciation ou des registres ${ }^{1}$ a été remplacée par la triade poésie/roman/théâtre, dont le caractère hétérogène saute aux yeux : le roman désigne un genre narratif, alors que le théâtre désigne un texte écrit pour la représentation scénique et que la poésie réfère plutôt à des traits sémantico-formels. La question du narratif, rapporté exclusivement au roman, devient difficile à penser en dehors de celui-ci alors que des notions comme celle de «théâtre épique»(Brecht) ou de «récit poétique» (étudié par Tadié) réintroduisent la question des registres. Néanmoins l'intérêt de cette triade est de nous obliger à partir, non pas de catégories explicatives établies a priori, mais de divisions empiriques, attestées par des collections et des inscriptions paratextuelles. Elle délimite un ensemble qui correspond au noyau dur de la littérarité, face à des textes à l'identité plus fluctuante, et elle invite par ses incohérences mêmes à distinguer les productions attestées des critères théoriques qui prétendront les décrire. Poéticiens et linguistes ne s'en sont pas privés mais, me semble-til, de façon souvent schématique. C'est pourquoi je voudrais à présent examiner les propositions les plus célèbres en montrant les apports et les difficultés qui en découlent.

\section{Les apports de Jakobson, Ruwet, Benveniste et Dominicy}

A une époque où institutionnellement la littérature et la linguistique restent plus séparées que jamais, il n'est pas inutile de souligner l'intérêt de linguistes éminents pour le discours poétique.

\subsection{Jakobson et Ruwet}

On sait que Jakobson, dans les Essais de linguistique générale, passe en revue les fonctions du langage découlant des six éléments constitutifs de la communication verbale, et définit la fonction poétique du langage comme « la visée (Einstellung) du message en tant que tel, l'accent mis sur le message pour son propre compte » $(1963: 218)$ en ajoutant aussitôt : 
Toute tentative de réduire la sphère de la fonction poétique à la poésie, ou de confiner la poésie à la fonction poétique, n'aboutirait qu'à une simplification excessive et trompeuse. (ibid.)

Il n'y a par ailleurs chez lui aucun aplatissement de la poésie sur le lyrisme : il signale l'importance de la fonction référentielle dans la poésie épique, de la fonction émotive dans la poésie lyrique et de la fonction conative dans la poésie "supplicatoire et exhortative». Mais ce qui lui importe, c'est de définir la fonction poétique, qui consiste, dit-il, à « projet[er] le principe d'équivalence de l'axe de la sélection sur l'axe de la combinaison » (p. 220):

En poésie, chaque syllabe est mise en rapport d'équivalence avec toutes les autres syllabes de la même séquence; tout accent de mot est censé être égal à tout autre accent de mot $[\ldots]$ les séquences délimitées par des frontières de mot deviennent commensurables, un rapport est perçu entre elles, qui est soit d'isochronie, soit de gradation. (p. 220-221)

Cette « réitération régulière d'unités équivalentes » combinée à la notion d' " attente frustrée » donne à la poésie son contour intonatif spécifique. Après avoir présenté plusieurs exemples de « modèles de vers », Jakobson consacre la suite du chapitre à l'examen des relations entre le son et le sens exemplifiées par la rime et les allitérations puis aux équivalences sémantiques et aux parallélismes syntaxiques en différents points de la séquence. Le poème apparait ainsi comme un ensemble complexe de ressemblances et de différences, ce qu'illustrera l'étude des « Chats » de Baudelaire, co-signée par Lévi-Strauss et publiée en 1962 dans la revue $L^{\prime} H o m m e^{2}$.

La réflexion de Jakobson comporte plusieurs acquis essentiels : la distinction entre poésie et fonction poétique est heureuse car elle rend bien compte par exemple du recours à la forme versifiée pour une affiche publicitaire. D'autre part, Nicolas Ruwet, en précisant la notion de parallélismes, montrera que ceux-ci affectent la structuration superficielle du message, et s'ajoutent aux facteurs habituels de cohésion, d'ordre sémantico-pragmatique, ou y suppléent, quand ceux-ci s'avèrent défaillants :

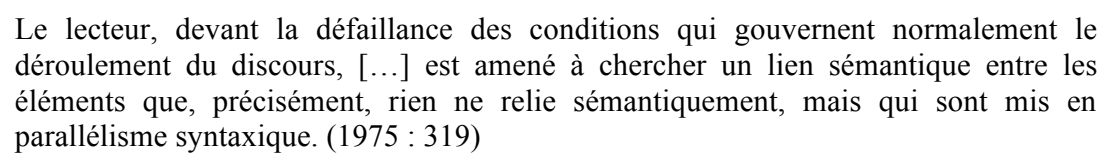

Ruwet éclaire ainsi les mécanismes de compréhension des textes poétiques en faisant droit à leur cohésion-cohérence à la fois très forte sur certains plans et lacunaire sur d'autres. Il montre en effet dans des analyses très fines que les parallélismes suscitent des processus d'évocation symbolique différents de la pensée conceptuelle. Il complète la théorie de Jakobson en précisant les relations entre forme et sens au sein du poème, ce qui lui permet d'opposer la «prose versifiée » dépourvue de tout effet symbolique à la vraie poésie (1975 : 349), mais aussi de récuser l'idée très répandue que la poésie serait une subversion radicale du langage ordinaire (cf. la note 1 p. 321).

Mais le premier volet de la théorie de Jakobson, à savoir l'autotélisme de la poésie, contenait des ambigüités qu'explicite très bien l'article d'Aroui (1996). Certaines avant-gardes dans les années 60-70 l'ont compris comme une suiréférentialité dont elles ont fait un critère absolu de littérarité : le langage littéraire est défini par un fonctionnement en boucle où le seul référent du texte est la littérature. Il s'agissait alors de lutter contre le primat donné à la représentation de la réalité dans les poétiques héritées d'Aristote, mais cela fut fait souvent de façon excessive en bannissant la fonction référentielle. Nous verrons plus loin que ce débat sur la référentialité de la poésie constitue un des enjeux majeurs de la réflexion sur la poésie et sur la littérature en général que le changement de contexte épistémologique permet sans doute de poser avec plus de justesse.

L'accent mis « sur le message en tant que tel » peut en fait se comprendre de façon moins forte comme le poids donné dans la poésie aux mots et aux énoncés en tant que tels, de sorte qu'ils constituent un élément essentiel du contexte permettant l'interprétation. Ce phénomène propre aux énoncés poétiques explique les difficultés de la traduction et résulte pour partie au moins des parallélismes. 
L'apport de Jakobson, enrichi par la réflexion théorique et les analyses textuelles de Ruwet, reste précieux pour comprendre la textualité spécifique du poème. D'autre part le recours de Ruwet aux concepts de symbolisme et d'évocation permet de progresser sur le plan de la sémantique de la poésie. Sur ce point en effet, la pensée de Jakobson reste prisonnière d'une dichotomie trop sommaire entre métonymie et métaphore, la première associée au roman, la seconde à la poésie ${ }^{3}$. Dans les Essais, Jakobson fait de l'ambigüité « un corollaire obligé de la poésie » mais il semble la définir essentiellement par la superposition de différentes interprétations et ne s'y attarde pas. Il aborde incidemment (p. 238239) la question de la référence et de l'énonciation pour dire qu'elles sont elles aussi ambigües car « dédoublées », sans justifier son affirmation. Ce qu'il dit du locuteur et du destinataire s'applique en réalité à tout énoncé littéraire, et la référence dédoublée est illustrée sans plus de détails par la formule des conteurs majorquins « cela était et n'était pas », qui renvoie plutôt à la fictionnalité. Il est vrai que dans ces années-là, les questions de référence et d'énonciation étaient encore fort peu étudiées par les linguistes. Notons en revanche que, malgré son attachement au structuralisme, Jakobson cherche à comprendre ce qui se passe aux deux pôles de la production et de la réception du poème. C'est une constante chez tous les linguistes qui travaillent sur la poésie que leur objet, quel que soit le moment épistémologique auquel ils appartiennent, les oblige à se poser la question de la relation destinateur / destinataire.

\title{
3.2 Benveniste
}

Benveniste, qui ne cite à aucun moment Jakobson dans son manuscrit ${ }^{4}$, n'en est pas très éloigné dans ses constats initiaux :

\begin{abstract}
A la différence du langage ordinaire, le langage poétique fait voir les choses en se faisant voir lui-même. Il est d'abord contrainte formelle, le schème <du vers $>$ préexiste au dire poétique, la mesure fixe, la répétition, le retour scandent le discours avant même qu'il soit énoncé. [...] Ce moule doit donc façonner préalablement les mots du discours. Voilà une première condition, externe. [...] La condition interne maintenant : ce sont les choses dites qui sont la poésie, non ce à quoi elles renvoient ou peut-on dire : les énonciations poétiques ne renvoient qu'à elles-mêmes? (p. 46 $6^{5}$ )
\end{abstract}

Benveniste répond un peu plus loin que le langage poétique réfère bien à quelque chose, mais que, voulant «nous faire éprouver son expérience <émotionnelle>» (p.50), il « iconise » en même temps qu'il « signifie ». Après avoir utilisé les verbes « représenter» et «évoquer» (p. 130), Benveniste opte pour « iconiser» dans son effort pour définir la référence dans le discours poétique. La question de la référence, sous-jacente à la visée du message en tant que tel, devient en effet centrale dans Baudelaire. Par iconisation, Benveniste cherche à définir une propriété du langage poétique qui consiste non pas à nommer l'émotion, mais à la faire ressentir. Là où la signification produit entre le destinateur et le destinataire un partage de type conceptuel, l'iconisation produit un partage de type expérientiel ${ }^{6}$ :

Les mots sont choisis et assemblés en vertu non d'une idée à énoncer, mais $<$ d'abord $>$ d'une émotion à rendre sensible, car c'est aux sens qu'ils s'adressent d'abord : c'est de l'émotion qu'émane l'idée en poésie. (p. 62)

Le signe linguistique est conceptuel ; l' "icône" poétique est chaque fois unique. [...] Le poète éveille le sentiment, éduque la perception, avive l'impression de la chose unique, du jamais-encore-perçu. Mais c'est lui qui crée cette émotion qu'il suscite, ce sont les mots qu'il a su assembler. (p. 600)

On pourrait penser qu'on a là l'opposition traditionnelle qui met la poésie du côté du sensible et le langage «ordinaire » (à quoi Benveniste oppose la poésie) du côté du rationnel. Vraisemblablement Benveniste est encore tributaire de cette vieille dichotomie, mais ses propos vont au-delà, car ce qu'il vise par l'idée, c'est un lien signifié / référent stabilisé collectivement, alors que l'émotion se trouve du côté d'une reconfiguration unique de ce lien par le travail sur le langage. Ceci est formulé plus clairement dans un autre passage :

Ainsi au rapport signifiant $\mid$ signifié $\mid$ référent le langage poétique ajoute (ou substitue) évocant $\mid$ évoqué $\mid$ émotion $<$ initiale>. (p. 138) 
L'important à mes yeux réside moins dans cette impulsion émotionnelle initiale où Benveniste voit le départ du poème ${ }^{7}$ que dans l'effort pour décrire un fonctionnement sémantique où les mots sont importants en eux-mêmes parce que seuls leur agencement et leurs correspondances peuvent faire ressentir au lecteur ce qui n'a pas encore été conceptualisé au niveau collectif. Dans ce processus, le sens dénotatif passe au second plan derrière les associations syntagmatiques et paradigmatiques que le mot « fait lever » (p. 260) :

En poésie il n'y a pas d'intenté-idée il y a un «sens » intérieur aux mots, qui est fonction de leur assemblage, (alors qu'en prose il y a une référence extérieure au discours, un monde dont le discours traite) auquel les signes renvoient). (p. 412)

Par exemple l'iconisant nuit étant lié par la rime à l'iconisant luit, l'iconisé NUIT acquiert par là même une certaine clarté (p. 134). Benveniste est ici très proche de Jakobson, et propose en quelque sorte une paraphrase explicative de sa définition de la fonction poétique.

Mais son étude prend aussi en compte d'autres éléments linguistiques, ce qui n'étonnera pas chez l'auteur des Problèmes de linguistique générale. Il s'intéresse à l'énonciation, notant que le discours poétique « ne s'adresse effectivement à aucun partenaire, mais à des entités. Dieu, la nature, le lecteur, l'aimée etc. » (p. 246). Lorsqu'il ajoute que « l'auteur du discours pose des questions qui n'attendent aucune réponse, sauf de lui-même, et qui doivent mettre le lecteur dans le même état d'interrogation et d'attente ", il décrit très bien les deux niveaux de l'énonciation: celui qui concerne l'auteur et son lecteur et celui qui concerne le locuteur du discours, lequel n'a pas vraiment d'interlocuteur (p. 238). Son étude des tiroirs verbaux qui dégage leur valeur contextuelle en s'appuyant sur l'ensemble du recueil est aussi très intéressante. Je reviendrai sur ces différents points lorsque je tenterai de proposer un programme pour l'étude linguistique de la poésie.

\subsection{Dominicy}

Si Benveniste, vers 1967, sans nier l'importance des relations paradigmatiques au sein du poème, met l'accent sur leurs effets sémantiques, c'est avec Marc Dominicy que la réflexion sur la sémantique en poésie va se développer mais à partir des prémisses posées par Jakobson. Son objectif est donc de décrire « l'action que les propriétés formelles »-phonologiques, morphologiques, syntaxiques et métriques - du texte poétique « exercent sur les mécanismes interprétatifs mis en œuvre, ou sollicités, par la réception ou la production d'un poème » $(1990: 10)$.

Il s'emploie tout d'abord à anéantir l'idée, encore présente chez ses devanciers, d'une opposition entre une langue ordinaire conçue comme un code et une langue poétique tantôt plus vraie, tantôt indéchiffrable. Il est à noter que Benveniste n'échappe pas à ce travers, même si le fait de barrer « langue » devant « poétique » et de remplacer le mot par «discours » témoigne de son insatisfaction (p. 246) devant ce binarisme. De fait Benveniste, s'il croit encore à une langue ordinaire, notionnelle, qui veut « convoyer un message », est bien conscient que la poésie est " une langue intérieure à la langue » (p. 132) et lorsqu'il écrit qu' « en poésie la distinction de la forme et du fond (à supposer qu'elle ait en soi un sens) est abolie », la parenthèse montre bien à son corps défendant l'inanité de la conception usuelle du sens dans la langue ordinaire. Mais il n'arrive pas à s'en défaire. Dominicy, lui, l'a résolument abandonnée au profit de quatre modalités sémantiques: logique, poétique, rhétorique, empirique, qui devraient permettre non pas de classer « toutes les productions textuelles attestées ou imaginables » mais « de saisir la spécificité d'un nombre assez restreint de types discursifs » (1989: 500). Quant au langage « ordinaire » ou «quotidien » - les guillemets sont de Dominicy - il est faux de croire qu'il «jouisse d'une sorte de transparence qui le sépare des discours où prédomine l'une des quatre modalités » (ibid.). Exit donc l'aporie résultant de l'opposition binaire «langue poétique / langue quotidienne ». Dans Poétique de l'évocation, les autres modalités sémantiques ne sont pas reprises, mais l'évocation poétique est envisagée comme un cas particulier d'évocation. Celle-ci est dans son ensemble opposée au traitement on line du langage par le récepteur mais envisagée comme une possibilité inscrite au cœur du fonctionnement du langage dans ses rapports avec la pensée. 
Dominicy récuse également l'idée que les textes logiques comme les textes poétiques n'auraient aucun rapport avec le réel, se borneraient à " "montre[r]" ou "reprodui[re]" la structure constitutive d'un univers dont $\mathrm{il}[\mathrm{s}]$ ne "di[raien]t" strictement rien » (1989: 501). Dans ces deux modalités « le réel est censé préexister au texte » et " ne se laisse pas identifier à l'interprétation éventuellement visée par l'auteur ou l'usager » (p.502). Il subsiste donc une indétermination irréductible quant à la réalité que le texte veut atteindre. Mais cela ne pose pas de problème car, selon Dominicy, les «modalités logique et poétique instaurent, entre le texte et le réel, une relation totalement indifférente aux difficultés soulevées par le vague et l'indécision » (p.505). Le vague est ici entendu comme la possibilité pour un énoncé de susciter des jugements contradictoires d'adéquation ou pas au réel - dire par exemple de tel meuble qu'il est grand ne garantit pas que tous soient d'accord -, et l'indécision comme le fait pour un locuteur de se trouver dans l'impossibilité de dire si un énoncé est valide ou pas, ce qui arrive par exemple quand on lit qu'une pierre est aveugle. A l'inverse, les modalités rhétorique et empirique s'emploient à gérer le vague et l'indécision par des techniques explicites (voir aussi 1994: 119-121). La définition de la modalité poétique s'insère donc dans une théorie d'ensemble de la production/interprétation des textes, et ce qui nous retiendra ici, c'est que le recours à l'évocation est le moyen pour la poésie de ne pas avoir à se préoccuper du vague et de l'indécision. En effet l'évocation repose pour Dominicy sur l'activation en mémoire «de représentations prototypiques partagées » qui permettent un certain flou d'un locuteur à l'autre. Ruwet explicite très bien ce flou à propos de la « Ballade des dames du temps jadis » :

Dans l'expérience de chacun, toute une série de choses, idées, perceptions, souvenirs, sont associées, d'une manière plus ou moins directe, plus ou moins précise, à la neige : la blancheur, le froid, le côté immaculé, le côté évanescent, la tristesse ou la stérilité, etc. De même toutes sortes de choses sont associées aux «belles dames du temps jadis » [...] Ces deux ensembles confus, qui varient plus ou moins d'un lecteur à l'autre $[\ldots]$ sont mis directement en rapport, projetés l'un sur l'autre ; des analogies entre eux, plus ou moins claires, sont suscitées. (1975:319-320)

Dans ce passage trop long pour que je puisse le citer entièrement, on comprend le lien entre les parallélismes superficiels caractéristiques du discours poétique et l'évocation. Celle-ci naît de la mise en relation par la syntaxe (le refrain interrogatif « Mais où sont les neiges d'antan? ») des dames du temps jadis et de la neige. Dominicy confirme ce lien entre la forme versifiée et le sens en affirmant que le vers « s'apparente [...] à la parole toute faite que constituent les adages, proverbes, expressions figées et tours idiomatiques ». Comme eux, le poète présente "le discours énoncé comme déjà dit, et donc pourvu d'une signification immédiatement accessible » (1989: 502) car stockée dans la mémoire à long terme. On peut dès lors parler de polyphonie dans la mesure où «le texte montre sa prise en charge par un énonciateur universel » (1994: 131). Par ailleurs la proximité entre texte poétique et genre épidictique (recours à l'amplification, énonciation universalisante, épithètes de nature) confirme que le poème prend appui sur des valeurs et des représentations partagées. Le travail fait par Dominicy sur les variantes de plusieurs poèmes (1996) ou sur la comparaison entre la version poétique et la version narrative d'un même évènement $(1992,1994)$ met en lumière cet effort de généralisation qui tend à susciter chez le lecteur des prototypes plutôt qu'à décrire une situation singulière.

Contrairement à Aroui (1996), il me semble que le lien entre les parallélismes et l'évocation est assez net dans la théorie de Dominicy, même s'il est vrai que c'est surtout dans Poétique de l'évocation que la formulation de ce lien se précise. Le livre de 2011, en effet, s'attache à montrer comment les déviations suscitées par la structure double du discours orientent l'interprète vers un traitement symbolique du message qui implique entre autres de s'appuyer sur une vérité sémantique présentée comme acquise pour construire une vérité représentationnelle dépendant du point de vue du locuteur. La structure double (logico-grammaticale et métrico-rythmique) entraine le déclenchement de deux processus évocatifs qui se déroulent en tandem, l'un traitant des percepts inanalysés que suscite l'organisation poétique, l'autre des contenus propositionnels extraits de l'organisation linguistique (2011:245). Marc Dominicy en conclut que l'évocation poétique vise au premier chef à créer ou réactiver des croyances stéréotypées, l'émotion éprouvée découlant du sentiment de reconnaissance procuré par le poème et non l'inverse. 
Poétique de l'évocation définit l'effet poétique par six facettes (79-145) : une forte cohésion textuelle, des parallélismes induisant des déviations syntaxiques ou sémantiques, des ruptures de pertinence au niveau local, un brouillage entre singularité et généricité, un accès complet à la singularité d'une expérience - ce qui fait dire que la poésie «traduit l'ineffable »-, en même temps qu'un défaut de communication. En envisageant le discours poétique dans ses propriétés à la fois structurelles, sémantiques et énonciativo-pragmatiques, Marc Dominicy élabore une théorie puissante qui constitue une avancée importante dans la réflexion linguistique de la poésie. Cela n'épuise pas pour autant ni les questions, ni le champ des recherches, comme je voudrais le montrer à présent.

\section{Sémantique, iconisation du signifiant et énonciation : trois composantes autonomes du texte}

Dans cette dernière partie, après avoir situé la réflexion sur la poésie dans le nouveau paradigme épistémologique créé par le développement des études linguistiques depuis quarante ans, je proposerai une catégorisation des textes en fonction de trois axes indépendants, définis en m'appuyant à la fois sur les théorisations antérieures et sur une longue pratique d'analyse de textes poétiques de genres et d'époques variés.

\subsection{Changements épistémologiques}

La façon dont nous envisageons à présent le rapport entre le langage et le réel amène nécessairement, sans renier leurs apports, à relativiser les thèses de Jakobson ou Benveniste. En effet, ce que nous savons maintenant du langage nous interdit d'opposer le discours poétique et le discours ordinaire. Le discours ordinaire a révélé toute sa complexité au fil des études dont il a été l'objet, qu'il s'agisse de sa structuration, des opérations de reformulation qui le jalonnent, de la négociation des rapports entre les interactants dont il est l'enjeu. Par ailleurs les travaux menés en analyse de discours, dans le droit fil du dialogisme bakhtinien, ont bien montré que tout discours construit son référent et comporte une certaine opacité à l'égard du réel que nous ne pouvons atteindre qu'indirectement, en nous appropriant ou en contestant les discours tenus antérieurement à son propos $^{8}$. La dimension référentielle est à la fois présente dans tout discours et complexe à analyser du fait des reformulations dont les savoirs et les croyances font constamment l'objet. De ce point de vue la différence entre la littérature (et en son sein la poésie) et les autres discours est, à mon sens, plus une différence de degré et d'intentionnalité que de nature. On trouve les prémices de la vision actuelle du référent dans un passage du Baudelaire :

\footnotetext{
La référence poétique est intérieure à l'expression et révélée peu à peu par cette expression qui la crée. Car la référence est <en poésie> une expérience <émotionnelle et personnelle>, au lieu qu'en langage ordinaire elle est l'objet hors du langage que le langage modèle et qui est en même [temps] posé comme objectif, approximé par le langage. (p.398)
}

Si l'on ne s'arrête pas à la dichotomie devenue intenable entre poésie et langage ordinaire, on constate que Benveniste ouvre ici une piste très intéressante sur la question de la subjectivation de l'objet du discours. D'un côté, en effet, il y a des discours qui posent le référent comme objectif même s'il est en réalité modelé par le langage, et de l'autre, des discours qui posent le référent sinon comme construit par leur activité même, du moins comme au point de contact entre le réel et le travail du locuteur.

Mon effort se situe donc dans le prolongement des travaux de Dominique Maingueneau sur le discours littéraire et vise à proposer des catégorisations qui permettent de penser la continuité entre les discours littéraires et les autres discours.

\subsection{Trois axes pour classer les textes}

Le premier axe de classement des textes est sémantique et concerne leur accessibilité pour un lecteur moyen ne disposant pas de compétences particulières ou au contraire leur forte opacité, leur décrochage 
par rapport aux échanges quotidiens. D'un côté figureraient les discours activant sans effort des représentations déjà communes parce qu'ils reposent sur des dénominations stabilisées et sont constitués d'énoncés facilement compris en raison de leur proximité avec d'autres énoncés déjà circulants; de l'autre se situeraient les discours où les référents des entités et des procès ne préexistent pour ainsi dire pas à l'énonciation qui les crée et où l'activité du co-énonciateur est fortement sollicitée pour coconstruire un nouvel objet de discours modifiant ses représentations discursives. Sur cet axe, les discours scientifiques qui construisent leur objet et le redéfinissent constamment seraient voisins des discours littéraires et se distingueraient de ceux-ci par les moyens mis en œuvre pour partager les représentations ainsi construites. On trouverait à l'opposé les proverbes, les discours quotidiens, et dans l'entre-deux les discours médiatiques. Cet axe se manifeste essentiellement sur le plan du lexique, des chaînes de dénomination, des relations cotextuelles et de la construction des isotopies. Je m'éloigne sur ce point de Dominicy en ce que, pour moi, l'évocation poétique ne consiste pas à créer ou activer des représentations stéréotypées, mais à produire de nouvelles représentations certes présentées d'emblée comme généralisables mais dont la nouveauté est soulignée par divers procédés à l'intérieur du message luimême.

Un deuxième axe concerne les propriétés intrinsèques du langage indépendamment de sa valeur de signe. Ces propriétés sont mises en sourdine à une extrémité de cet axe et mises en relief à l'autre extrémité . Cette mise en relief correspond en gros à la fonction poétique jakobsonienne, ou à l'iconisation du signe décrite par Benveniste. Elle s'appuie sur le fait que les propriétés phoniques et syntactico-rythmiques du langage sont susceptibles de produire par elles-mêmes des émotions et des significations qui ne découlent pas du référent visé par le discours, même si elles peuvent être en adéquation avec lui. À une extrémité de cet axe se situeraient tendanciellement les discours qui cherchent à construire une représentation discursive en n'ayant pas d'autre souci dans l'agencement des mots et des énoncés que celui de la clarté et de l'efficacité de l'expression. On y trouverait les textes informatifs, la plupart des textes philosophiques ou scientifiques, une bonne part des discours quotidiens et médiatiques. Le signe ici s'abolit au profit du référent. À l'autre extrémité se trouverait le discours poétique qui fait de cette dimension iconique sa quête première et dont le sens résulte pour une part plus ou moins grande de la mise en œuvre des parallélismes décrits par Jakobson et Ruwet. Il faudrait toutefois ajouter à ces moyens traditionnels en poésie des faits de disposition et de typographie qui se sont développés depuis la fin du XIXe siècle et qui peuvent être dominants dans une poésie faite pour être lue plus que dite. Entre ces deux pôles se situeraient tout un éventail de productions textuelles qui attachent de l'importance à cette dimension iconique mais la subordonnent à d'autres préoccupations: l'efficacité persuasive, la dynamique actionnelle, la dimension ludique. Les œuvres d'éloquence, maints textes publicitaires, les jeux de mots, ainsi que la littérature narrative ou dramatique occuperaient ainsi cet entre-deux, avec une position plus proche de l'un ou l'autre pôle selon les textes.

Un troisième axe, énonciatif, concerne le statut de l'énonciateur du texte par rapport au sujet empirique qui le produit et à la communauté discursive dans laquelle il se situe. Le classement des textes sur cet axe s'efforce de mesurer la façon dont l'énonciateur construit une scénographie plus ou moins autonome par rapport aux contraintes de la situation de communication ${ }^{9}$. On observe en effet que, dans une grande majorité de textes, la distinction théorique entre le sujet empirique producteur du texte et le locuteur qui assume la responsabilité des propos tenus est effacée. Même lorsqu'on sait qu'un homme politique fait écrire ses discours par un conseiller, rien de cette dissociation n'apparait dans le texte. Juridiquement l'auteur qui publie un essai portant son nom sur la couverture est tenu pour responsable des propos qui y sont tenus. L'écart toujours présent entre l'être-du-monde, pour reprendre les termes de Ducrot (1984 : 199 ) et le locuteur construit par le texte, écart théorisé par l'opposition en argumentation entre l'éthos préalable et l'éthos discursif (Amossy 2005 : 70), de même que l'écart symétrique entre l'auditoire réel et l'auditoire construit par le texte (ibid.: 36-37), sont rarement thématisés par les textes qui simulent une parfaite identité entre les communiquants et les énonciateurs, entre la situation de communication et la scène d'énonciation. Or, les textes littéraires fictionnels ont ceci de spécifique qu'ils exhibent cet écart en donnant la parole à des énonciateurs dont on sait qu'ils ne sont pas les producteurs empiriques du discours, et en créant un monde référentiel dont le caractère complètement ou partiellement imaginaire est reconnu par le lecteur. À cet égard, la poésie lyrique se distingue assez nettement d'autres genres 
littéraires, y compris poétiques, en ce qu'elle assimile la première personne du discours à l'auteur et le destinataire au lecteur tout en procédant à une décontextualisation des énoncés qui en permet une réception très différée par rapport aux circonstances intiales de production. Cette spécificité de la poésie lyrique a été bien perçue quoique théorisée de façon contestable par Hamburger (1986). Contrairement au théâtre et au roman qui incluent très souvent dans le texte même des références à leur double niveau énonciatif (l'auteur et le lecteur ou le public d'une part, les narrateur/narrataire et les personnages d'autre part), la poésie lyrique s'aligne sur le dispositif autobiographique qui postule une identité de l'auteur, du je narrant et du je narré (Lejeune 1975). Cet effet de transparence énonciative (Monte 2010 a) rejoint les discours ordinaires alors que, par ailleurs, le travail sur les niveaux sémantique et iconique éloigne la poésie de ces discours. C'est de cette tension que nait souvent le plus gros effet à réception de la poésie.

La question de l'énonciation est abordée aussi par la praxématique qui, dans ses travaux sur l'actualisation discursive (Barbéris 2001), propose une tripartition entre une subjectivité implicite (instance anonyme), émergente (instance explicite englobant le tu et le je) et pleinement réalisée (opposant le je et le $t u$ ). Cette vision dynamique de la subjectivation semble pouvoir rendre compte de phénomènes importants dans la poésie (Monte 2011). Mais l'articulation entre cette approche énonciative et celle qui concerne la manifestation ou le masquage de l'opération de construction de la scène énonciative reste à faire.

La typologie de Bronckart (1995) qui distingue les textes selon leur mode d'ancrage, impliqué ou autonome, et selon leur rapport aux coordonnées de l'action langagière (opposition entre le mode de l'exposer et le mode du raconter), est également de type énonciatif, et très opératoire pour distinguer des architypes de situations d'énonciation. Mais elle ne prend pas en compte la question du décrochage entre situation de communication et scène d'énonciation. Par ailleurs de nombreux textes se révèlent hétérogènes, passant par exemple du discours théorique au discours interactif ou cumulant les caractéristiques des deux discours, ou incluant du récit interactif dans du discours interactif., ou du discours interactif dans de la narration Très utile pour repérer des changements au sein d'un texte, la typologie de Bronckart ne me parait donc pas susceptible de permettre un classement des discours au niveau de généralité que je m'efforce d'atteindre (niveau de la scène englobante ou générique).

C'est pour la même raison que je ne propose pas de classer les textes sur des critères énonciatifs tels que l'homogénéité vs l'hétérogénéité des voix et des points de vue, ou bien l'affichage de la subjectivité vs l'effacement énonciatif. Dans de nombreux genres, appartenant à des formations discursives variées, l'énonciateur a certes le choix entre affirmer sa singularité ou, au contraire, éviter tout ce qui ressortirait à un éthos singulier et présenter son discours comme une on-vérité, pour reprendre les termes de Berendonner (1981: 62). Ceci vaut pour les discours ordinaires, politiques, philosophiques et bien sûr littéraires. Dans la littérature narrative, l'opposition entre des récits dont l'énonciateur intervient fréquemment dans la narration et des récits où il s'efface le plus possible s'avère tout à fait importante. On pourrait aussi pour le théâtre distinguer les pièces où l'auteur s'adresse au spectateur (fût-ce par la voix d'un porte-parole, coryphée ou prologue) de celles où il s'en abstient. Les travaux sur les genres des médias ou du discours philosophique (Moirand 2007, Rabatel 2004 entre autres) montrent également la pertinence d'une opposition entre discours à énonciation subjectivisée et discours à énonciation objectivisée. Mais il semble qu'il n'y ait que le discours médiatique où cette opposition fonde une différence générique, car, dans cette formation discursive, toutes les exceptions au principe de neutralité doivent être dument enregistrées et légitimées par un genre spécifique (billet, chronique, critique). Dans d'autres scènes englobantes, ces différences de positionnement énonciatif relèvent plutôt de profils d'énonciateurs, ou d'éthos discursifs, qui varient d'un texte à l'autre, de sorte qu'il ne me semble pas possible de fonder une typologie des textes sur ce critère. Certes les phénomènes analysés à la suite de Bakhtine sous le terme de dialogisme nous permettent de mieux comprendre le fonctionnement des textes - j'ai montré ailleurs (Monte 2010 b) que la poésie, contrairement à ce que dit Bakhtine (1978: 107), peut elle aussi incorporer une grande hétérogénéité énonciative - mais ils sont à prendre en compte dans un deuxième temps, une fois qu'a été analysée la façon dont le texte règle la relation entre instances empiriques impliquées dans la production effective du texte et coénonciateurs impliqués dans le fonctionnement énonciatif de ce même texte. À cet égard, la décontextualisation opérée par la poésie 
devra être distinguée de celle opérée par les discours théoriques, comme le manifeste un usage différent des tiroirs verbaux et des personnes.

À mon sens, l'axe énonciatif tel que je l'ai défini est aussi important que les précédents en ce qu'il permet notamment de distinguer les textes relevant de ce que Genette (1991) appelle la littérarité constitutive (roman, poésie, théâtre) de ceux qui relèvent de la littérarité conditionnelle. Toutefois l'opposition fiction / diction interne à la littérarité constitutive mérite d'être retravaillée en faisant intervenir les trois axes que je viens de définir. C'est ce que j'esquisserai à la fin de cet article. Mais auparavant, je voudrais ajouter que l'axe énonciatif rend compte également de la spécificité de textes à finalité non pas littéraire mais ludique, tels que les blagues ou les devinettes (souvent à la première personne) et de certains textes publicitaires mettant en scène des énonciateurs fictifs. La perception de la dissociation entre sujet parlant et locuteur s'avère essentielle dans certains genres discursifs alors que d'autres postulent son effacement.

\subsection{Gradualité et interrelation des critères}

Ce modèle, nécessairement grossier et sans nul doute amendable ${ }^{10}$, présente plusieurs intérêts. Tout d'abord, il distingue trois séries de phénomènes souvent confondus quand on parle de poésie :

- $\quad$ la formulation de l'objet de discours et le travail sur les représentations ;

- $\quad$ l'iconisation du signifiant ;

- le statut de l'énonciateur.

Les séparer conduit à un nouveau regard sur les classifications. Ainsi les discours scientifiques et poétiques ont-ils en commun de faire évoluer nos représentations par un travail proprement sémantique tout en se distinguant bien sûr sur les deux autres axes. Les archi-genres de la triade littéraire sont dans leur ensemble à l'un des pôles de l'axe 3 (distinction marquée du sujet empirique et de l'énonciateur) mais se distinguent sur les axes 1 et 2 où la poésie est sur une position plus extrême que le roman ou le théâtre. Les discours publicitaires et les histoires drôles partagent certains traits des textes poétiques sur les axes 2 et 3 mais pas sur l'axe 1. Le gain de cette approche multi-critères ne serait pas négligeable si elle conduisait à abandonner des typologies trop binaires.

La prise en compte simultanée des trois critères éclaire aussi sans l'absolutiser la différence entre roman et théâtre d'une part, poésie d'autre part. Globalement, on peut sans hésiter affirmer que la bonne réception des textes relevant de l'un ou l'autre de ces trois archi-genres requiert du récepteur la compréhension de la ficticité de leur scène énonciative : il est essentiel pour bien les interpréter de comprendre que les personnages qui s'adressent à nous ne sont pas réels, que le narrateur n'est pas l'auteur, que le locuteur/énonciateur premier s'émancipe de la personne de chair et d'os qui l'a créé ${ }^{11}$ et du contexte dans lequel le texte prend naissance, enfin, que le lecteur idéal proposé par le texte ne coïncide pas a priori avec les lecteurs réels que nous sommes. Il est possible cependant de recevoir un poème comme directement émis par un sujet parlant identifié au locuteur. Les erreurs d'interprétation qui en résulteront seront apparemment moins rédhibitoires que si l'on avait affaire à un roman ou à une pièce de théâtre. De même, le roman et le théâtre ont beau être des objets esthétiques, le travail sur la dimension iconique du langage y est d'intensité variable selon les époques, les auteurs, les esthétiques. Je garde donc l'opposition genettienne fiction / diction mais en la déplaçant quelque peu puisque, pour moi, la fiction a trait avant tout aux instances énonciatives et seulement par corollaire au monde représenté. Sur l'axe de la fiction, le roman et le théâtre font par essence intervenir des énonciateurs fictifs, alors que certaines formes de poésie peuvent faire croire à l'empiricité de leurs énonciateurs. Inversement, sur l'axe de la diction, la poésie comme archi-genre se définit par un usage nécessairement iconique du langage alors que le roman et la pièce de théâtre peuvent attacher plus ou moins d'importance à cette dimension iconique. Mais il faut éviter toute simplification excessive : envisager ces trois archi-genres à l'aune d'un seul critère ne permettrait pas de comprendre des pans entiers de la production littéraire : théâtre en vers, roman poétique, poésie épique, poésie d'intervention. 
Un tel modèle permet aussi de rendre compte de différences historiques au sein d'un archi-genre sans renoncer pour autant à une classification générale. Étant graduel il permet de positionner un (archi)genre de façon variable selon les époques sur tel ou tel des trois axes. Dans le cas de la poésie, il est clair, par exemple, que le travail sur les représentations est plus fort dans la poésie du XXe siècle que dans la poésie de la Pléiade ou la poésie romantique qui s'appuient davantage sur des lieux communs, propres à toute une communauté discursive. En revanche l'iconisation du signifiant est plus élevée - ou tout au moins plus visible - dans maints exemples de poèmes du XVIe, XVIIe ou XIXe siècles que dans des poèmes du XXe siècle non rimés et non métriques et à la syntaxe assez ordinaire. L'interaction entre l'axe 1 et l'axe 2 permet peut-être aussi d'avancer dans la question de la différenciation entre de bons et de mauvais poèmes. En effet, comme le notait déjà Ruwet, dans les mauvais poèmes, les parallélismes sont déliés de tout effet d'évocation et la poésie devient pure rhétorique - je prends ici le terme " rhétorique » dans son sens courant et non dans le sens spécifique que lui donne Dominicy -. Plus généralement, l'ajout à la structure logico-sémantique de parallélismes phoniques, rythmiques, métriques, syntaxiques est essentielle à la poésie, mais elle ne suffit pas à la caractériser. Seule la prise en compte des faits sémantiques et énonciatifs peut la distinguer par exemple de certains messages publicitaires. Proposer trois axes de différenciation permet de prendre en compte ce phénomène sans utiliser le terme trop général de «fonction poétique ».

La façon dont j'ai défini le troisième axe, en distinguant l'acte de communication et la mise en scène énonciative, évite l'assimilation indue de la poésie à l'expression des sentiments d'un sujet empirique. La poésie comme les autres genres littéraires construit une scénographie que le texte dans son avancée va légitimer. La dissociation sujets empiriques / énonciateurs peut y être niée (cas de certains poèmes lyriques), éludée (poèmes aphoristiques ou caractérisés par un effacement énonciatif) ou retravaillée (cas de la poésie satirique qui place souvent le satirisé en position d'énonciataire mais peut aussi construire un auditoire de connivence avec l'énonciateur contre une cible commune). Elle peut aussi y être évidente comme dans la poésie épique. Au-delà de la très grande variabilité des positionnements, la décontextualisation et l'effacement de la relation primaire auteur-lecteur affirment le rôle clé de la scénographie dans ce discours institué (au sens que Maingueneau 2007 : 75 donne à ce mot) très sensible à l'évolution du contexte historique et culturel.

Les trois axes, comme je l'ai indiqué plus haut, ne sont sans doute pas complètement indépendants. On peut penser par exemple que la modification des représentations ordinaires est facilitée par le travail d'iconisation du signifiant qui agit sur les expériences sensorielles et la mémoire du lecteur. C'est tout l'effort de Marc Dominicy que de montrer comment les parallélismes conduisent le lecteur à une interprétation symbolique différente de nos processus cognitifs usuels. Par ailleurs la construction d'un éthos discursif spécifique est à la fois facilitée et contrecarrée par l'iconisation du signifiant: si la métrique impose une organisation extérieure au discours et peut créer un éthos plus collectif qu'individuel, le choix des mots à la rime, les accords ou désaccords entre syntaxe et vers, les accents prosodiques singularisent la voix du locuteur/énonciateur et lui confèrent une très forte identité corporelle, qui fait passer au second plan le sujet empirique au profit des instances énonciatives. Le récepteur tendra d'autre part selon le dispositif énonciatif choisi à rapporter la représentation discursive à des énonciateurs identifiés, au producteur empirique, voire, comme le pensent certains (Philippe 2005) au texte lui-même. La transformation d'un texte en objet esthétique résulte vraisemblablement de la conjonction de facteurs appartenant à chacun des trois axes. Il y a là encore matière à de nombreuses recherches.

Notons par ailleurs que ces trois axes ne disent rien quant à la textualité locale : celle-ci, à mon avis, relève des constructions socio-historiques que sont les genres au sens strict ainsi que des projets individuels des énonciateurs, et ne peut être envisagée au niveau des archi-genres, sous peine d'exposer les analystes à des généralisations hasardeuses et au cinglant démenti des textes eux-mêmes. C'est ainsi que la question du degré de narrativité de la poésie ${ }^{12}$, très intéressant en soi, ne doit pas à mon sens être envisagé en général mais au cas par cas : il ne se pose pas de la même façon dans une poésie épique et dans une poésie lyrique, ni au XXe siècle comme au XVIe siècle. Il en va de même du statut de la description en poésie, ou de la question de l'autonomie du poème. Il est certain qu'en poésie la relation de 
la partie et du tout ne se pose pas de la même façon que dans un roman ou une pièce de théâtre, mais l'autonomie du poème isolé ne vaut que pour certaines productions textuelles. Dans l'épopée antique ou la geste médiévale, on peut isoler des morceaux de bravoure, mais à peu près de la même façon qu'on isole le récit par Rodrigue du combat contre les Maures ou le dîner offert par Hamilcar aux mercenaires dans Salammbô. A cet égard, le choix par Hugo de composer La Légende des siècles sous la forme d'une succession de poèmes réellement indépendants indique qu'il ne s'agit plus là d'une épopée au sens strict $^{13}$. La présence ou pas d'une chute dans les sonnets ou dans les poèmes en prose, les phénomènes d'ouverture et de clôture, de dominance d'une séquence - au sens qu'Adam (2011 : 161-164) donne à ce mot - sur une autre sont évidemment très importants, mais ne concernent pas la poésie comme archigenre. De même, la présence ou l'absence du vers, son caractère métrique ou non métrique, sont, selon moi, des faits de textualité locale, qui jouent un rôle essentiel dans la nature des parallélismes mis en œuvre dans le poème et dans sa caractérisation historique, mais qui ne sont pas définitoires de la poésie. Tout au plus pourra-t-on dire que, sous le rapport de l'axe 2, un poème en prose ou un poème en vers non métriques présente un déficit de parallélismes d'un certain type par rapport à ce qui a été la norme durant de longs siècles. Il s'agira alors d'observer si ce déficit est compensé par d'autres formes d'iconisation du signifiant ou par un travail spécifique sur les axes 1 et 3 qui permette d'identifier le texte comme poème. L'étude de Dernière heure de Cendrars par Marc Dominicy est à cet égard exemplaire de la méthode à suivre et démontre brillamment l'appartenance du texte à l'archi-genre poésie.

Je conclurai brièvement en disant que s'il est encore possible et légitime de parler de la poésie comme archi-genre, cela ne peut se faire qu'en élaborant une définition de la poésie qui ne repose pas exclusivement sur des phénomènes historiquement situés. Pour prendre en compte la forte évolution subie par cet archi-genre au fil des siècles, il faut se garder par exemple de proposer des définitions qui ne marchent bien que pour la poésie versifiée ou qui identifient la poésie au lyrisme et en excluent le narratif. Dans cet effort, les travaux des linguistes s'avèrent très stimulants car ils s'attachent moins aux genres historiques qu'à des traits généraux, bien que leurs exemples révèlent souvent une limitation au moins inconsciente du corpus. Mais jusqu'à une époque récente, leur réflexion, quoique toujours riche d'enseignements, a été entravée, même chez Jakobson qui a pourtant proposé une définition large de la fonction poétique, par une certaine incapacité à dépasser l'opposition binaire peu opératoire entre langage ordinaire et langage poétique. La théorie de Marc Dominicy est exempte de ce travers et peut constituer une base solide pour penser la poésie d'un point de vue linguistique. J'ai souhaité présenter à côté du sien un autre modèle, nettement plus empirique et encore en cours d'élaboration, mais qui se propose de situer la poésie par rapport aux autres discours littéraires - ce que Dominicy ne fait pas - et la littérature au sein des discours sociaux, dans une optique d'analyse du discours plus que de sémantique cognitive et en donnant une part importante à la question de la scène d'énonciation. La discussion est ainsi ouverte sur l'intérêt d'une approche modulaire par rapport aux approches unitaires qui ont prévalu jusqu'alors.

\section{Références bibliographiques}

Adam, J.-M. (2011, 3e éd. revue). La linguistique textuelle. Paris : Armand Colin.

Amossy R. (2000). L'argumentation dans le discours. Paris : Nathan (nouvelle édition 2006, Armand Colin).

Aron P., Saint-Jacques D. et Viala A. (2004). Dictionnaire du littéraire. Paris : PUF.

Aroui J.-L. (1996). L’interface forme/sens en poétique (post) jakobsonienne. Langue française, 110, 4-15.

Bakhtine M. (1978) trad fr [1987]. Esthétique et théorie du roman. Paris : Gallimard, coll « Tel».

Barbéris J.-M. (2001). Articles « Subjectivité dans le langage », "Subjectivité en même vs en soi-même », in Termes et concepts pour l'analyse de discours, Détrie C., Siblot P. \& Verine B. (éd.). Paris : Champion.

Benveniste E. (2011). Baudelaire (éd. établie par C. Laplantine). Limoges : Lambert-Lucas.

Berendonner A. (1981). Éléments de pragmatique linguistique. Paris : Minuit.

Bronckart J.-P. (1996). Activité langagière, textes et discours. Paris-Lausanne : Delachaux et Niestlé. 
Combe Dominique. (1989). Poésie et récit Une rhétorique des genres. Paris : José Corti.

—. (1992). Les genres littéraires. Paris : Hachette Supérieur.

Charaudeau P. et Maingueneau D.(dirs) (2002). Dictionnaire d'analyse du discours. Paris : Seuil.

Degrés $\mathrm{n}^{\circ}$ 111. (2002). Poésie et narrativité, Bruxelles.

Dominicy M. (1988). Y a-t-il une rhétorique de la poésie ?». Langue française, 79, 51-63.

—. (1989). De la pluralité sémantique du langage. Rhétorique et poétique. Poétique, 80, 499-514.

—. (1990). Prolégomènes à une théorie générale de l'évocation, in Sémantique textuelle et évocation. Vanhelleputte. M (dir). Louvain-la-Neuve : Peeters, 9-37.

-. (1992). Pour une théorie de l'énonciation poétique, in Enonciation et parti pris. W de Mulder. F Schuerewegen et L Tasmowski (éds). Amsterdam /Atlanta : Rodopi, 129-142.

—. (1994). Du style en poésie, in Qu'est-ce que le style ? Molinié G. et Cahné P. (éds). Paris : PUF, 115-137.

- (1996). La fabrique textuelle de l'évocation Sur quelques variantes des Fleurs du Mal. Langue française 110, 3347.

—. (2011). Poétique de l'évocation. Paris : Garnier.

Ducrot O. (1984). Le dire et le dit. Paris : Minuit.

Dürrenmatt J. (2005). Stylistique de la poésie. Paris : Belin.

Gaudin-Bordes L. et Salvan G. (2008). Les registres. enjeux stylistiques et visées pragmatiques. Louvain-la-Neuve : Academia Bruylant.

Genette G. (1979). Introduction à l'architexte. Paris : Seuil.

—. (1991). Fiction et diction. Paris : Seuil.

Guerrero G. (2000). Poétique et poésie lyrique.Essai sur la formation d'un genre. Paris : Seuil.

Hamburger K. (1986, trad.fr. $2^{\mathrm{e}}$ édition révisée en allemand : 1968). Logique des genres littéraires. Paris : Seuil.

Jakobson R. (1963, trad.fr.). Essais de linguistique générale I : Les Fondations du langage. Paris : Minuit.

—. (1977, trad.fr.). Huit questions de poétique. Paris : Seuil.

Lejeune P. (1975). Le pacte autobiographique. Paris : Seuil.

Maingueneau D. (2004). Le discours littéraire. Paris : Armand Colin.

-. (2007, $2^{\mathrm{e}}$ éd.). Analyser les textes de communication. Paris : Armand Colin.

Moirand S. (2007). Les discours de la presse quotidienne. Observer, analyser, comprendre. Paris : PUF.

Monte M. (2010 a). Auteur, locuteur, éthos et rythme dans l'analyse stylistique de la poésie, in Stylistiques ?, Bougault L. et Wulf J. (dir.). Rennes : PUR, 325-342.

-. (2010 b). Scénographie mouvante et hétérogénéité des points de vue dans L'Âge de l'Humanité d'André Salmon », in André Salmon, poète de l'Art vivant, Monte M. (dir.), Faculté de Lettres de 1'Université du Sud Toulon-Var, p.193-214.

-. (2011). Intersubjectivité et coénonciation dans la poésie contemporaine, in L'actualisation de l'intersubjectivité : de la langue au discours, Verine B. et Détrie C. (éds). Limoges : Lambert-Lucas, 119-138.

Philippe G. (2005). Le style est-il une catégorie énonciative ? in De la langue au style, Gouvard J.-M. (dir.), Lyon : Presses universitaires de Lyon, 145-156.

Rabatel A. (2004). Stratégies d'effacement énonciatif et posture de surénonciation dans le Dictionnaire philosophique de Comte-Sponville. Langages, 156, 18-33.

Ruwet N. (1975). Parallélismes et déviations en poésie, in Langue, discours, société, Kristeva J. et al. (éds). Paris : Seuil, 307-351.

Schaeffer J.-M. (1989). Qu'est-ce qu'un genre littéraire? Paris : Seuil. 
Tadié J.-Y. (1978). Le récit poétique. Paris : PUF.

\begin{abstract}
${ }^{1}$ Le Dictionnaire du littéraire définit les registres comme des «catégories de représentation et de perception du monde que la littérature exprime et qui correspondent à des attitudes en face de l'existence, à des émotions fondamentales » (p.529). Le lyrique, l'épique, le tragique et le comique sont considérés traditionnellement comme des registres. Gaudin-Bordes et Salvan (2008) soulignent la dimension pragma-énonciative des registres et la complexité de la notion.
\end{abstract}

${ }^{2}$ Pour une critique de cette lecture, cf. M. Riffaterre, Essais de stylistique structurale, Flammarion, 1971, p.307-364.

${ }^{3}$ Faute de place, je ne développerai pas cet aspect de la pensée de Jakobson qui est développé dans Questions de poétique.

${ }^{4}$ Sauf erreur de ma part, on ne trouve aucun emploi de « parallélisme » dans le manuscrit sur Baudelaire. Benveniste ne tient visiblement pas à afficher une quelconque dette vis-à-vis de Jakobson dont on sent pourtant l'influence sur sa réflexion.

${ }^{5}$ Je prends le parti de ne pas indiquer les fins de ligne par des barres obliques, contrairement à l'édition de Chloé Laplantine, car cela hache l'énoncé et ne me parait pas essentiel pour mon propos. Je note en revanche par $<>$ les mots ajoutés au-dessus de la ligne et par des italiques les mots soulignés.

${ }^{6}$ Cette insistance sur l'expérience rejoint les réflexions d'Hamburger sur le je lyrique, mais là où Hamburger réfléchit sur l'ancrage énonciatif des énoncés, Benveniste se pose la question de leur effet.

${ }^{7}$ Il nuance d'ailleurs cette opinion en écrivant p.604 que « le poète part d'une sensation vive, d'une perception aigüe, parfois même d'un schéma vide rempli seulement d'un désir de rythme»

${ }^{8}$ On trouvera un bon résumé de ces positions aux articles «Objet de discours » et «mémoire discursive» du Dictionnaire d'analyse de discours (2002).

9 À la suite de Charaudeau et Maingueneau (2002) - articles «situation de communication» et «scène d'énonciation »-, je distingue situation de communication et scène d'énonciation.

10 En ce qui concerne le premier axe, l'articulation avec les quatre modalités sémantiques de Marc Dominicy reste à faire, si tant est que les cadres théoriques soient compatibles. Plus que la question de la gestion du rapport au réel sous l'angle du vague et de l'indécision, c'est en effet celle de la familiarité des propositions énoncées qui a retenu mon attention.

${ }^{11}$ Sur la question du locuteur du poème, on pourra lire le chap. 1 de Dürrenmatt (2005) et Monte 2010 a.

${ }^{12}$ On consultera avec intérêt à ce sujet le numéro 111 de la revue Degrés.

${ }^{13}$ Sur le maintien de l'épopée au XXe siècle, on lira avec profit Désirs et débris d'épopée au XXe siècle, (2009), Neiva S. (éd.), Berne : Peter Lang. 